\title{
Infrared absorption cross sections, and oscillator strengths of interstitial and substitutional double donors in silicon
}

\author{
S. G. Pavlov $\odot,{ }^{1}$ L. M. Portsel $\odot,{ }^{2}$ V. B. Shuman, ${ }^{2}$ A. N. Lodygin $\odot,{ }^{2}$ Yu. A. Astrov $\odot,{ }^{2}$ N. V. Abrosimov $\odot,{ }^{3}$ \\ S. A. Lynch $\odot,{ }^{4}$ V. V. Tsyplenkov, ${ }^{5}$ and H.-W. Hübers ${ }^{1,6}$ \\ ${ }^{1}$ Institute of Optical Sensor Systems, German Aerospace Center (DLR), 12489 Berlin, Germany \\ ${ }^{2}$ Ioffe Institute, Russian Academy of Sciences, 194021 St. Petersburg, Russia \\ ${ }^{3}$ Leibniz-Institut für Kristallzüchtung (IKZ), 12489 Berlin, Germany \\ ${ }^{4}$ School of Physics and Astronomy, Cardiff University, CF24 3AA Cardiff, Wales, United Kingdom \\ ${ }^{5}$ Institute for Physics of Microstructures, Russian Academy of Sciences, 603950 N. Novgorod, Russia \\ ${ }^{6}$ Institut für Physik, Humboldt-Universität zu Berlin, 12489 Berlin, Germany
}

(Received 19 August 2021; accepted 20 October 2021; published 29 November 2021)

\begin{abstract}
Infrared absorption cross sections and corresponding oscillator strengths of several intracenter transitions of double donors in silicon, interstitial magnesium $(\mathrm{Mg}$; group IIA) and substitutional chalcogens $(\mathrm{Ch}=\mathrm{S}$; Se; group VI), were determined for impurity densities in the ranges $1 \times 10^{14}-1.6 \times 10^{15}$ atoms $/ \mathrm{cm}^{3}$ for $\mathrm{Mg}$ and $2 \times 10^{13}-2 \times 10^{16}$ atoms $/ \mathrm{cm}^{3}$ for chalcogens. The concentrations of electrically active atomic and diatomic donor centers were derived from the Hall effect measurements. The experimental integrated cross sections were obtained from low-temperature impurity absorption spectra. The oscillator strengths of related donor transitions were derived and compared with those for shallow single donors in silicon, both determined experimentally and predicted theoretically. The transitions of oscillator strengths of double donors follow the decreasing trend with decreasing radius of donor ground states and increasing an impurity binding energy.
\end{abstract}

DOI: 10.1103/PhysRevMaterials.5.114607

\section{INTRODUCTION}

Novel impurity centers in silicon are interesting because of the valuable insights they can reveal about fundamental material physics, and for their technological exploitation potential. Excited group- $\mathrm{V}$ substitutional donors in silicon, such as phosphorus or arsenic, are solid-state analogs of the hydrogen atom and share many common quantum properties [1]. The spatial extent of the donor electron wave function extends several nanometers from the donor site to overlap thousands of silicon unit cells, so that the wave function provides a probe of the local host crystal environment [2]. The elemental group-IV crystal hosts (diamond, silicon, and germanium) are also unique because of the absence of polar optical phonons. This removes an important scattering mechanism, and it is this unique property that many research groups are ultimately trying to exploit for quantum technology applications $[3,4]$. The double donors in silicon have an even richer spectroscopy in comparison to the hydrogenlike single donors. Neutral double donor atoms (both interstitial and substitutional) behave as solid-state analogs of the helium atom. Furthermore, if one of the double donor electrons is captured at a neighboring acceptor site, then a different hydrogenlike spectral signature corresponding to the ionized double donor can be observed [5], which may be implemented for development of spin-photon interfaces [6]. Substitutional (S, Se, and Te) and interstitial $(\mathrm{Mg})$ double donor atoms in silicon also show a tendency to cluster together to form diatomic centers, and also to cluster together with vacancies, and these complex crys- tal defects manifest as additional distinct spectral signatures $[5,7]$.

The binding energies of the double donor centers and their complexes lie in the range $30-600 \mathrm{meV}$, so that midinfrared photons with sufficient energy can promote electrons from the donor ground state into the conduction band where they can be detected electrically. Technological applications which could harness this potential include pyrometry and environmental sensing. Multiwavelength pyrometers combine the midinfrared spectral information from several data points to reconstruct the blackbody curve to give an accurate remote measurement of temperature $[8,9]$. The vibrational spectra of many atmospheric pollutants also lie in this so-called fingerprint region of the electromagnetic spectrum. For example, the $2614.6 \mathrm{~cm}^{-1}$ (324.2 meV) symmetric stretch of the $\mathrm{H}_{2} \mathrm{~S}$ molecule lies a few meV above the minimum energy required to ionize the neutral sulfur donor $(319 \mathrm{meV})$. Midinfrared silicon-based sensors $[10,11]$ would be more desirable than the alternative $\mathrm{HgCdTe}$-based technology from the point of view of toxicity, as well as due to inherent compatibility with complementary metal-oxide semiconductor (CMOS) architectures [12].

One useful property of electrically active impurity centers (EAICs) is that relatively simple resistivity measurements can be used to establish their concentration, and this procedure is routinely carried out in industry for single-electron centers. The accurate determination of EAIC density becomes critical for cases where multiple centers contribute to the conductivity. The partial contributions of such centers can be weighted, 
but this requires knowledge of their relative abundances. This issue can be solved by infrared spectroscopy, enabling direct determination of EAIC concentrations using calibrated absorption coefficients. Such calibrations were made for several conventional impurities in elemental semiconductors: substitutional single-electron group- $\mathrm{V}$ donors and group-III acceptors in silicon and germanium [13-15], and substitutional boron acceptor in diamond [16]. Integrated absorption coefficients and cross sections can be used to derive oscillator strengths $f$ of intracenter transitions and then compared with theoretical calculated values for several substitutional hydrogenlike centers in silicon and germanium $[17,18]$.

While the impurity spectra of single hydrogenlike donor centers in silicon and germanium were described theoretically $[13,18]$, showing good enough qualitative (energy spectra) and quantitative (calibration factors and oscillator strengths of intracenter transitions) agreement with experiment, a quantitative theoretical description of two-electron (double) centers in silicon is still missing. However, there is a substantial body of experimental data in the literature about double donors in silicon. The infrared spectra of neutral double donors reveal the para-series of heliumlike transitions with the structure of dipole-allowed transitions ending in odd-parity excited states $(1 s \rightarrow n p)$, in most details similar to those of the hydrogenlike donors. In addition to atomic donors $(\mathrm{Ch})$, diatomic centers $\left(\mathrm{Ch}_{2}\right)$ as well as donor complex $\mathrm{Ch}_{c} X$ centers determine structures of infrared spectra of chalcogens $(\mathrm{Ch}=\mathrm{S}, \mathrm{Se}, \mathrm{Te})$ in $\mathrm{Si}[5]$. To explain similarity in the $1 s \rightarrow$ $n p$ structure of transitions, the authors [19] assumed-for deep double substitutional donors in $\mathrm{Si}$ - the strong screening of an impurity ion by the inner electron remaining in the "one-particle" ground state, and by other core electrons. We followed such an assumption for calculation of donorstate wave functions and the strength of related intracenter transitions (see below). A similar effect is supposed to play a role for magnesium $(\mathrm{Mg})$, which serves as an interstitial double donor in Si [20]. The concentration broadening of the spectral lines relating to double substitutional and interstitial donors in $\mathrm{Si}$ is also very different [5,21], which remains unexplained so far. Thus empiric calibrations of optical transitions of double donors are an important issue, not only for easy determination of EAIC concentrations, but also for the future theoretical investigations of such centers in elemental group-IV semiconductors to investigate their potential for quantum photonics and technology $[4,22]$.

In this work, infrared cross sections and oscillator strengths for several intracenter transitions of atomic and diatomic double donors, interstitial magnesium $(\mathrm{Mg})$ and substitutional chalcogens, sulfur $(\mathrm{S})$ and selenium $(\mathrm{Se})$, were determined from impurity infrared absorption spectra. Calibration coefficients were found for the strongest intracenter transitions observed at studying samples that satisfy the main criteria for accurate determination of constants, which are optically thin samples and reduced interference features. The oscillator strengths $f$ of intracenter transitions were compared with the theoretically estimated values, obtained in the simplified model of a double donor in silicon. Clear trends of decreasing $f$ with decreasing radius of a donor ground state and increasing an impurity binding energy follow those found for a hydrogenlike donor in $\mathrm{Si}$ but show significantly stronger dependences on these key parameters.

\section{DIFFUSION DOPING AND SAMPLE PREPARATION}

Doping by diffusion is the main technology used for production of silicon crystals with moderate concentrations of EAIC double donors. In the present study, dopants were introduced from a solid or gas phase at high temperatures; see Supplemental Material [23] for details of the diffusion doping and obtained parameters of investigated samples [24-27]. A large number of samples have been characterized to improve the degree of statistical confidence, in addition to ascertaining whether different diffusion conditions and postdiffusion treatment exhibit detectable influence on the mean values of oscillator strengths of impurity transitions. Most of the wafers in these experiments were float-zone grown (FZ) p-type silicon with low concentrations of shallow acceptors.

\section{A. Si:Se, Si:S}

Selenium and sulfur donors were formed in samples by the high-temperature diffusion from the dopant gas phase in sealed quartz ampoules using the regimes optimized in the former studies [24,25]. Wafers with a typical thickness of $1.0 \mathrm{~mm}$ and diameter of $30 \mathrm{~mm}$ were placed in a quartz ampoule together with a weighed portion of the high-purity $(99.9999 \%)$ elementary selenium/sulfur. The vapor pressure of a dopant ( $p_{\mathrm{Se}}$ in the range of $0.027-0.87$ bar for Se and $p_{S}=0.01-1.0$ bar for $\mathrm{S}$ ) at a diffusion temperature was determined by the chalcogen mass in an ampoule. The amount of a dopant necessary for obtaining the target concentration of EAIC was calculated in the ideal gas approximation. Then an ampoule with samples and a dopant was filled with argon (Ar) gas and sealed. The argon partial pressure in a sealed ampoule was estimated to ensure that the gas pressure did not exceed 1 bar at the diffusion temperature. Typically, the diffusion process was performed at a temperature of $1240{ }^{\circ} \mathrm{C}(\mathrm{Se})$ and $1200{ }^{\circ} \mathrm{C}(\mathrm{S})$ over the course of 240-264 h (Se) and 24-72 $\mathrm{h}$ (S). The higher the pressure of a dopant gas phase in the volume of a sealed volume at the diffusion temperature, the larger is the concentration of atomic chalcogen EAIC and also accompanying diatomic EAIC and impurity complexes. Postdiffusion treatment, such as annealing (A) and/or annealing plus quenching (AQ), of a doped sample modifies the partial concentrations of atomic and diatomic chalcogen EAIC [24-27]. Annealing at high temperature results in increased concentration of atomic $\mathrm{Ch}$ centers, while the concentration of diatomic $\mathrm{Ch}_{2}$ centers is reduced. Rapid quenching "freezes" the obtained high density of Ch centers in silicon [27].

The opportunity to use such a postdiffusion treatment of chalcogen-doped samples for obtaining the dominating either atomic or diatomic double donors ("end members") offers a unique option for the accurate assessment of individual centers and serves as an internal reference for the crystals with the mixed donor content.

After diffusion has been performed, the top $\sim 10 \mu \mathrm{m}$ of the sample surface was removed by mechanical grinding and then optically polished with a typical wedge of $0.5^{\circ}-1.5^{\circ}$ between the polished facets. 


\section{B. Si:Mg}

Magnesium donors were formed by the high-temperature diffusion from the solid phase in sealed ampoules using the "sandwich" technique [28] optimized in recent studies $[29,30]$. Mg (of purity up to $99.999 \%$ ) thin layers were deposited on the surfaces of a $\mathrm{Si}$ wafer by thermal evaporation of the metal in vacuum. The wafer was then "sandwiched" between two other Si wafers, and the "sandwich" was heated in a sealed quartz ampoule in an argon atmosphere. The typical diffusion temperature was between $1100^{\circ} \mathrm{C}$ and $1250{ }^{\circ} \mathrm{C}$, and the diffusion time between $1 \mathrm{~h}$ and $7.5 \mathrm{~h}$. To suppress the decomposition of the solid-state phase of interstitial magnesium in a sample, the ampoule was cooled in the stream of nitrogen following the diffusion step. In some experiments, a rapid cooling was applied - through dropping an ampoule with a sample into a mineral oil. It was found, however, that this method of cooling did not change the concentration of atomic $\mathrm{Mg}$ EAIC, as compared to the above case of the relatively slow cooling, but modified content of the shallow Mg-related centers [7], which appear on a very low level relative to main $\mathrm{Mg}$ EAIC.

After diffusion, the welded outer Si wafers were mechanically ground back to the original interface layer of the central wafer and then a further $\sim 30-50 \mu \mathrm{m}$ depth. The Si:Mg samples that were prepared to measure the light absorption in the IR range had final thickness in the range of $1-2 \mathrm{~mm}$ with polished facets wedged to $1.0^{\circ}-1.5^{\circ}$. Thus the applied technique of doping enables bulk samples to be prepared with a relatively homogeneous distribution of interstitial atomic $\mathrm{Mg}$ with concentrations up to $1.5 \times 10^{15} \mathrm{~cm}^{-3}$.

\section{APPROACHES FOR CALIBRATIONS OF DOPANT CONCENTRATION AND INTEGRATED ABSORPTION AT INTRACENTER DONOR TRANSITIONS}

The main approach to finding the calibration coefficients relates to empirical determination of integrated absorption coefficients of particular intracenter transitions, as well as the use of known concentrations of a particular EAIC, derived from an alternate analytical technique. Most of previously performed calibrations of single-electron impurity centers in silicon relied on derivation of concentration of their dominant dopant by a four-probe measurement of room-temperature resistivity, which is well known and tabulated in international databases, e.g., Ref. [31]. While applicable for shallow centers, this approach cannot be directly applied for double electron centers, since (particularly for chalcogen impurities in silicon) there can be coexistence of atomic ( $\mathrm{Ch})$, diatomic $\left(\mathrm{Ch}_{2}\right)$, and complex $\left(\mathrm{Ch}_{c} X\right)$ electrically active centers, characterized by overlapping ranges of their absorption spectra [19] and by common ranges of temperature-dependent changes in concentrations of their charged states.

Hall effect measurements were used in the present study to derive concentrations of electrically active centers with different thermal ionization energies. This was achieved through the analysis of temperature-dependent concentrations of free electrons $n(\mathrm{~T})$, similar to the approach applied in Ref. [24] for doubly charged sulfur donor centers.
Low-temperature infrared absorption spectra of samples were taken with two Fourier-transform spectrometers (Bruker Vertex 80v), see for details Ref. [20]. The calibrated absorption spectra show the relevant spectral features associated with the electrically active impurity centers and indicate the strength of intracenter transitions, as well as some additional infrared-active spectral features related, e.g., to local vibrational modes of oxygen.

\section{A. Determination of dopant concentration by temperature-dependent Hall effect}

Hall effect measurements in the temperature range of $T=78-500 \mathrm{~K}$ for the samples doped with chalcogens and 78-300 K for $\mathrm{Si}: \mathrm{Mg}$ samples were used to determine concentrations of electrically active donor centers. This was performed by fitting a calculated temperature dependence of a free electron concentration $n(\mathrm{~T})$ to the experimental data points for a given sample (see Supplemental Material [23] for further details). A trial function $n(\mathrm{~T})$ was calculated through the numerical solution of the electroneutrality equation for an $n$-type semiconductor [24], taking into account both free electrons and charged localized centers (donors and acceptors). As trial parameters in a calculation of $n(\mathrm{~T})$, the ionization energies of neutral (0) and ionized (+) states $\mathrm{Mg}, \mathrm{S}$, and Se centers in silicon were derived from infrared absorption spectroscopy.

Typical examples of the $n(\mathrm{~T})$ in silicon samples doped by chalcogens and magnesium are shown in Fig. 1, while the derived concentrations are given in the Supplemental Material [23] [Tables 1(Mg), 1(S), 1(Se)]. Partial contributions in the $n(\mathrm{~T})$ dependences for centers with different ionization energies are shown by individual solid curves. Such a curve illustrates a change in the filling of an impurity energy state as the temperature is varied. The "saturation" in a curve above a specific temperature indicates a completed thermal ionization of a particular center. The larger the thermal ionization energy, the higher is the saturation temperature range of a center. We note that the deepest centers possess large saturation temperatures, above those available in the setup, and were not reached in the experiments. The technological parameters of diffusion doping, a $\mathrm{Ch}$ vapor pressure in the course of a diffusion and postdiffusion thermal treatment with subsequent quenching of samples, hint at the ratios of the achieved partial concentration, so that the doped samples with large deviations from the mean values can be selected out for the follow-up spectroscopic calibration.

For chalcogen double donors $\left(D D^{0}\right)$, thermal activation energies of atomic and diatomic (molecular) centers are different enough (factor 1.48-1.70) to be clearly differentiated in the Hall temperature dependences [Figs. 1(a) and 1(b)]. Singly ionized diatomic $\mathrm{Ch}_{2}{ }^{+}$centers have thermal activation energies close to those of atomic neutral $\mathrm{Ch}^{\circ}$ centers (factor 1.16-1.27) but occur at relatively low concentrations due to low acceptor residual concentrations in the investigated samples. There were no intracenter transitions related to $\mathrm{Se}_{2}{ }^{+}$ centers observed in the infrared low-temperature absorption spectra. Therefore their contribution in the slopes of temperature Hall dependences and related corrections to the derived $\mathrm{Ch}^{\circ}$ concentrations was disregarded. The appearance of 
(a)

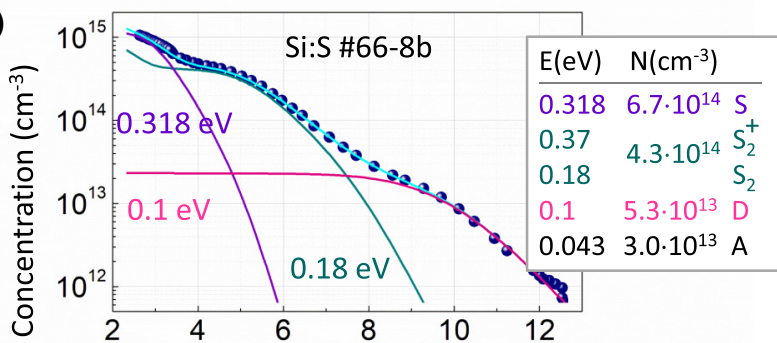

(b)

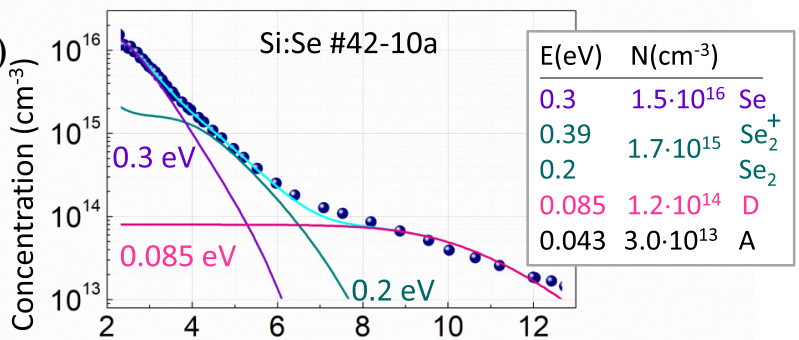

(c)

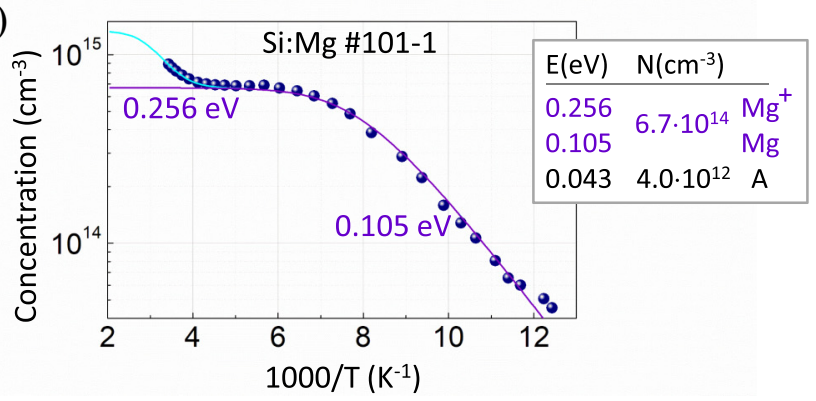

FIG. 1. Examples of temperature dependences of a free electron concentration in Si samples doped by chalcogens and magnesium. The dots are experimental data, the solid lines are calculated fitting curves. The lowest (black) concentration values in all tables on the right side indicate the residual dopant in the original Si crystals, before the diffusion doping. D, A stay for shallow (single-electron) donor and acceptor centers, respectively.

centers with the activation energies about of $0.1 \mathrm{eV}$ [Figs. 1(a) and 1(b)] was attributed to $\mathrm{Ch}_{c}(X)$ complexes, confirmed by infrared spectra of the Si:Ch samples, having ionization energies between 0.082 and $0.116 \mathrm{eV}$ [5]. The initial acceptor/donor concentration in all Si crystals was residual and did not contribute noticeably to the evaluated concentration of double donors in the neutral phase $\left(D D^{0}\right)$.

In several $\mathrm{Si}: \mathrm{Mg}$ samples, additional Mg-related complexes [7] could be responsible for the $n(\mathrm{~T})$ slope at the lowest temperatures in the Hall measurements. We have made further treatment of the values of $\mathrm{Mg}$ density, derived from the $n(\mathrm{~T})$ slope, in order to estimate the influence of Mg-related complexes, see the Supplemental Material [23] for details. As we found, the corrections to the concentration of $\mathrm{Mg}^{0}$, obtained in the model with and without inclusion of $\mathrm{Mg}-\mathrm{O}$ centers [activation energy $E\left(\mathrm{Mg}-\mathrm{O}^{0}\right)=0.125 \mathrm{eV}$ [32]], does not exceed $10 \%$, while corrections due to double $\mathrm{Mg}^{*}$ center [activation energy $E\left(\mathrm{Mg}^{* 0}\right)=0.094 \mathrm{eV}$ [21]] do not exceed $20 \%$ (see the Mg densities, derived from the Hall measurements and the corrected values, in the Supplemental Material [23], Table I (Mg)). These values are comparable to the accuracy of obtaining the Hall effect data and the $n(\mathrm{~T})$ fitting procedure. In both cases, the presence of the $\mathrm{Mg}$ complexes brings a systematic lowering of the neutral $\mathrm{Mg}^{0}$ donor concentration $N_{\mathrm{Mg} 0}<N_{\text {Hall }}$. The corrections into the $\mathrm{Mg}^{\circ}$ center concentration improve the standard deviation of the integrated absorption for $\mathrm{Mg}^{\circ}$ centers (see Supplemental Material [23]) but can be also avoided with a judicious small change in the accuracy for the mean $N_{\mathrm{Mg} 0}$ value, if a few $\mathrm{Si}: \mathrm{Mg}$ samples with the large (15\%-25\% in total) estimated concentration of the Mg-related complexes were disregarded.

Nevertheless, quantitative data sets were obtained for a large ensemble of samples with different parameters-that is, for samples prepared from initial silicon wafers with different concentrations of residual impurities. Averaging scattered experimental data over the whole set of studied samples give nearly the same values (under 10\% accuracy) of the calibration factor and oscillation strength, which are observed in the samples prepared from a high-purity silicon which provide low concentrations of $\mathrm{Mg}-X$ complexes.

Most of the values derived from infrared spectra integrated absorptions were used for the calculation of transition oscillator strengths and calibration factors for double donors. A few strong outliers were disregarded in these calculations.

\section{B. Integrated absorption from infrared impurity absorption spectra}

As a rule, the optical calibration is valid over a certain range of impurity concentrations $N$, where integrated absorption coefficient of impurity intracenter transitions, taken as an integral under the absorption spectrum $\alpha(v)=$ $\mathrm{N} \sigma(v)\left(\mathrm{cm}^{-1}\right)$ over the shape of a particular line of the absorption, $v_{12}\left(\mathrm{~cm}^{-1}\right)$ - that is, for the transition between states 1 and 2-remains linear to the EAIC concentration, holding a constant integrated absorption cross section $\sigma_{i}$ :

$$
\alpha_{i}=\int_{\text {line }} \alpha(v) d v=N \sigma_{i}\left[\mathrm{~cm}^{-2}\right] .
$$

A few conditions should be obeyed when performing and analyzing the absorption spectra.

The spectral resolution and the spot size of diagnostic light were adapted in our experiments to obtain the correct line profile at different concentrations and sample forms. The observed spectra of chalcogen donors have FWHM $\approx$ $0.6-2.5 \mathrm{~cm}^{-1}$ for different $\mathrm{Ch}$ and $\mathrm{Ch}_{2}$ concentrations. The spectra of magnesium-doped samples had FWHM $\approx$ $0.5-1.1 \mathrm{~cm}^{-1}$ for different $\mathrm{Mg}$ concentrations. Most of spectra were taken at a resolution of $0.1-0.3 \mathrm{~cm}^{-1}$, which varied when studying low- and moderately doped samples. For a few highly doped thin samples $(\sim 500 \mu \mathrm{m})$, where a standard $1^{\circ}$ wedge was mechanically challenging at $7-10 \mathrm{~mm}$ sample sizes, the spectral resolution was reduced up to $1 \mathrm{~cm}^{-1}$, while the IR light spot size was reduced to $1.5 \mathrm{~mm}$ in order to minimize the collection of interfered light onto detector and to minimize intensity of related fringes in the lower-frequency spectral range. The residual interference has been reduced by the Fourier band block filtering. The absence of sidebands in an interferogram of light transmitted through a sample [see inset in Fig. 2(a)] is an appropriate test of low impact of the light interference inside the sample on the result of a measurement. 

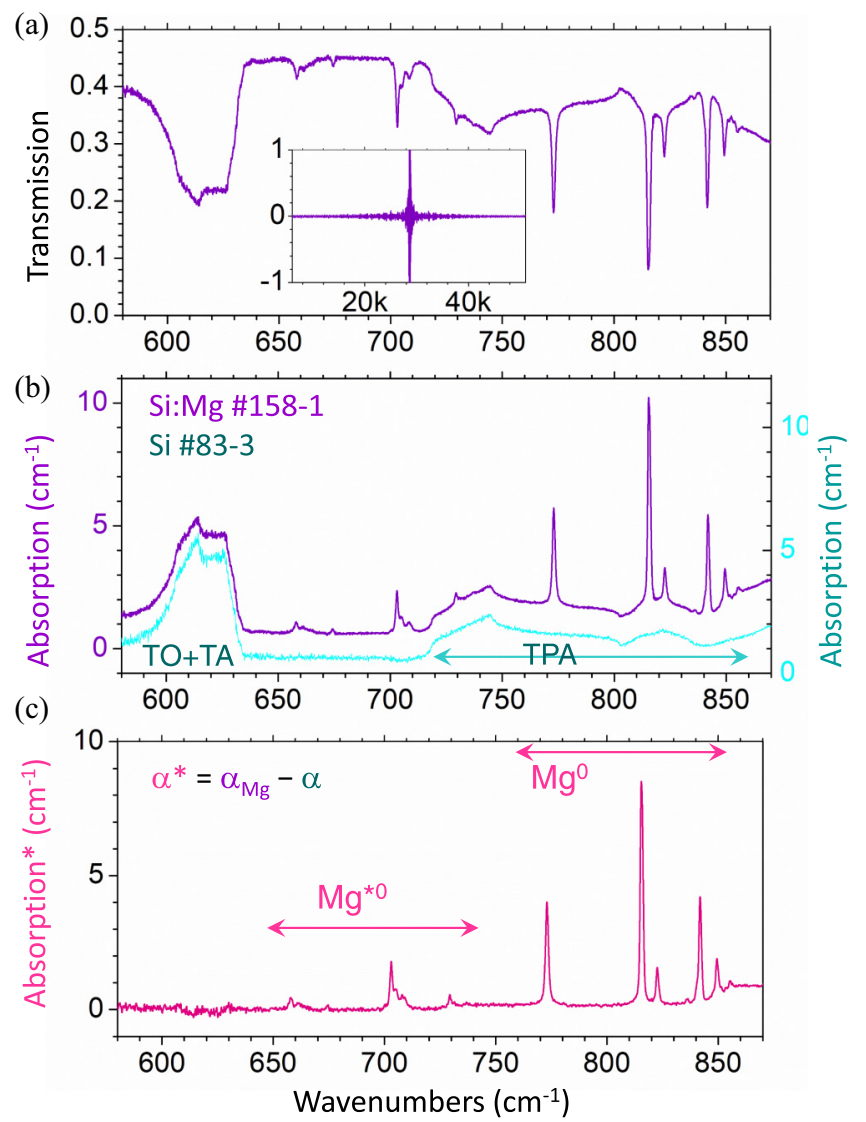

FIG. 2. An example of treatment of a sample absorption spectrum for reduction of the two-phonon absorption band (TPA, > $700 \mathrm{~cm}^{-1}$ ) overlapping with $\mathrm{Mg}$ neutral donor intracenter transitions in $\mathrm{Si}$ : (a) transmission spectrum of the $\mathrm{Si}: \mathrm{Mg}$ no. 158-1 sample. Inset: Interferogram of the spectrum; (b) absorption spectra of Mg-doped and undoped Si samples, shifted for a better view; (c) a difference spectrum of the Si:Mg sample after subtracting the spectrum of undoped $\mathrm{Si}$.

A transmission spectrum of light $T_{S}(v)$ should reflect an optical path of diagnostic light through a sample that has an appropriate analytical expression: for instance, approaching a single path propagation in a wedged sample that describes transmission as

$$
\frac{T_{s}(v)}{T_{0}(v)}=[1-R(v)]^{2} \exp [-\alpha(v) L]
$$

where $T_{0}(v)$ is the reference spectrum, taken through the same optical path without a sample, $R(v)$ is the reflection from the sample surface, and $L$ is the sample mean thickness. Since low-temperature dispersion of $\mathrm{Si}$ in the spectral range of interest does not exceed $2 \times 10^{-4}$ [31], we used a constant value of $n_{\mathrm{Si}}=3.398(1)$ as a low-temperature infrared $(4-13 \mu \mathrm{m})$ refractive index of silicon [33,34], which yields $R(v) \approx 0.2973$.

The total infrared absorbance in (2) through an investigated sample should satisfy the Beer-Lambert law, i.e., $\alpha(v) L<1$, thus maintaining a linear relationship between light attenuation and concentration of absorbing centers. This condition has been managed for most of the cases [see Table(s) 1, Supplemental Material [23]]. We controlled the level of transmitted light at impurity resonances [Fig. 2(a)] and used

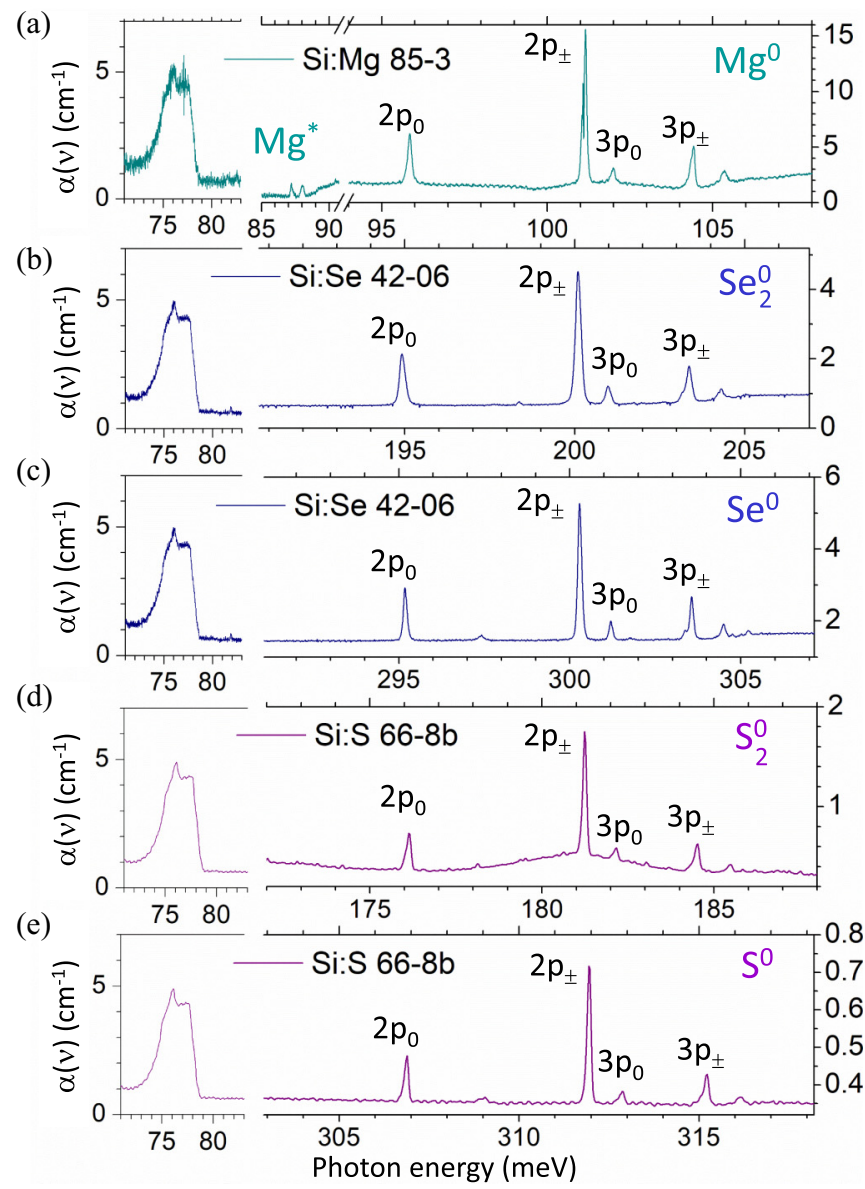

FIG. 3. Examples of calibrated absorption spectra for samples with multiple donor centers in Si: (a) Si:Mg no. 85-3, neutral $\mathrm{Mg}^{*}$ and $M g^{0}$ double donors; (b), (c) Si:Se no. 42-06, neutral atomic and diatomic selenium double donors; (d), (e) Si:S no. 66-8b, neutral atomic and diatomic sulfur double donors.

spectra for calibration where transmission exceeds a value of at least $6 \%$, thus satisfying the necessary conditions for "an optically thin sample" at the selected transitions.

The absorption spectra were derived by (2) and calibrated to a distinct spectral feature [Figs. 2(b) and 3], a $\mathrm{TO}(\mathrm{X})+\mathrm{TA}(\mathrm{X})$ two-phonon absorption (TPA) band [35]. This spectral range occurs at the photon energies fairly remote from the lines of interest and does not overlap with any intracenter transitions of double donors or impurity photoionization bands. Such a calibration of infrared absorption is necessary due to unavoidable day-to-day, small variations of the light intensity in the optical scheme and the detector response of the individual spectrometer, instrumental differences between used spectrometers, as well as due to individual light refraction in the wedged $\mathrm{Si}$ samples. At low and moderate concentrations of double donors the line shapes of intracenter transitions are Lorentzian-like [Fig. 5(f)], while at above $10^{16} \mathrm{~cm}^{-3}$ the shapes for $D D$ transitions start to change towards a Gaussian type together with significant $(>50 \%)$ linewidth broadening. This also causes a reduction of the defined absorption cross sections of transitions that is so strong for several of the deepest chalcogen states that the OD for such samples no longer increases together with a donor 
concentration [see, e.g., the sample Si:Se \#44-4, Table I (Se), Supplemental Material [23]].

At these concentrations the characteristic ratio of integrated absorptions for the transitions ending in the $2 p_{ \pm}$ and $2 p_{0}$ states, remains, however, relatively stable, which is similar to observations for the shallow donors [15]. When absorbance in the samples exceed $1.1(\alpha L>1.1)$, the integrated absorption for such transitions deviates from the values observed in dilute doped crystals. At low absorbance values $(\alpha L<0.01)$ the absorption in lines exhibit the widest scattering of values [Figs. 5(a) and 5(b)].

We checked the difference between a few approaches to derive the integrated absorption for the intracenter transitions overlapping with TPA bands in silicon and occurring at phononic band slopes. One approach was to eliminate the TPA bands by subtracting the spectrum of the undoped Si sample (no. 83-3), which has passed the same heating process as the Si:Mg samples [Fig. 2(c)]. Another option was the Bruker OPUS software tool accounting for the background slope in the integration of the line area. We also applied an internally developed code to calculate the integrated absorption. We found a tiny difference, below $1 \%$, between such approaches, which is about the same as observed from the calibration of experimental spectra, which is based on the TPA absorption band. Finally, we derived the integrated absorption by the last two methods, while the difference between them was used in calculation of the error bar.

Also, the overlapping of intracenter transitions close in energy [such as $1 s\left(A_{1}\right) \rightarrow 4 p_{0}$ and $1 s\left(A_{1}\right) \rightarrow 3 p_{ \pm}$in Si:Se] in moderately doped samples was evaluated using the sums of Gaussian- or Voigt-modeled line shapes. The derived integrated absorptions were, as a rule, far off the mean calibration dependence, and corresponding data were finally disregarded. The dependences of integrated absorption for the transitions into the $2 p_{0}$ (or) and $2 p_{ \pm}$states of double donors (Fig. 4) enable determination of the calibration factors (inversed integrated absorption cross sections) $\sigma_{i}^{-1}$ for the investigated impurity centers (Tables I-V).

\section{Oscillator strengths of intracenter transitions from impurity absorption spectra}

Characteristic values of the EAIC intracenter transition strength, such as oscillator strength $f_{12}$ and transition dipole matrix element $\mu_{12}$, can be directly derived from the measured integrated absorption cross sections $\sigma_{i}$ using the following relationships:

$$
f_{12}=\frac{n_{S i} m^{*}}{\pi r_{e}} \sigma_{i} \quad \text { and } \quad \mu_{12}^{2}=\frac{3}{2} \frac{g_{2}}{g_{1}} \frac{\hbar \varepsilon_{0}}{\pi^{2} v_{12}} \sigma_{i},
$$

adapted from Refs. [36,37]; here $m^{*}=3 m_{0} /\left(1 / m_{l}+2 / m_{t}\right) \approx$ 0.26 , where $m_{0}, m_{l}$, and $m_{t}$ are masses of a free electron, effective longitudinal, and transverse masses of electrons in the Si conduction band, respectively; $r_{e}=2.818 \times 10^{-13} \mathrm{~cm}$ is the classical electron radius, $g_{2}$ and $g_{1}$ are the degeneracy factors of the final and initial states involved in the optical transition $1 \rightarrow 2$, $\hbar$ is the reduced Planck constant, $\varepsilon_{0}$ is the electric permittivity of vacuum, and $v_{12}$ is the frequency of a transition $\left(\right.$ in $\mathrm{cm}^{-1}$ ).
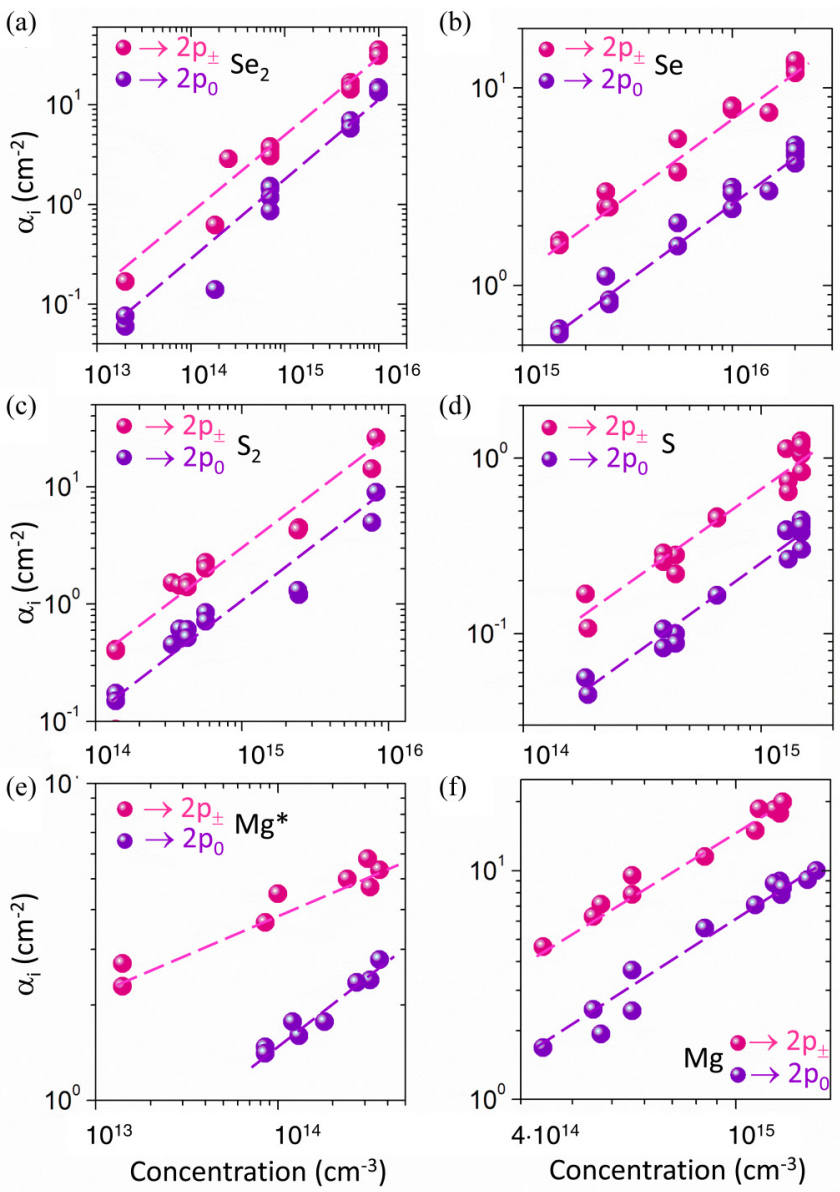

FIG. 4. Experimental dependences of integrated absorption for the two most intense intracenter transitions (from the ground state into $2 p_{0}$ and $2 p_{ \pm}$states) of neutral double donors in $\mathrm{Si}$ on concentrations of the centers (a), (b) selenium-doped Si; (c), (d) sulfur-doped $\mathrm{Si}$; (e), (f) magnesium-doped Si. Note that the concentration of the $\mathrm{Mg}^{*}$ center was estimated using the general trend for the oscillator strength dependences on the double donor transition energy [see Figs. 5(c) and 5(d)] and using either sum of multiple transitions into the $2 p_{0}$ or $2 p_{ \pm} \mathrm{Mg}^{*}$ states. It is given for the reference purpose only, not for the accurate derivation of the $\mathrm{Mg}^{*}$ parameters. The dashed lines are the best fit by eye to the experimentally derived data points.

Cross sections for the intracenter transitions of double donors $\sigma(v)=\alpha(v) / N_{\mathrm{DD}}$ were obtained from the measured peak values of absorption $\alpha(v)$ at frequencies corresponding the $D D$ transitions and concentration $N_{\mathrm{DD}}$ of particular double donors derived from the Hall measurements data and calculations that followed. The calibration factors were calculated for the integrated absorption values with weak dependence on the estimated EAIC concentrations, that is, those ranges as plotted in Fig. 4. Spectral resolution in the experiments for the obtained data is $0.016 \mathrm{meV}\left(0.13 \mathrm{~cm}^{-1}\right)$. The accuracy of determination of the transition energy is $\pm 0.016 \mathrm{meV}$.

The obtained results are collected in the Tables I-V together with the calculated values for the transitions $f$ obtained within the double donor model as described below, in Sec. IV. The "end members" (dominating atomic $\mathrm{Ch}^{0}$ or dominating diatomic $\mathrm{Ch}_{2}{ }^{0}$ donors) of the chalcogen-doped crystals cluster 
TABLE I. Absorption cross sections and oscillator strengths for most intense intracenter transitions of neutral Mg double donor in silicon. The transition energy and cross-section values are averaged over data for investigated samples, with optical density for a transition, $\alpha L<1.1$. The accuracy of weak transitions into the $\mathrm{Mg}^{0}$ state on the slope (!) of the stronger adjacent line is $\pm 0.025 \mathrm{meV}$.

\begin{tabular}{|c|c|c|c|c|c|c|}
\hline \multirow[b]{2}{*}{ Donor } & \multirow{2}{*}{$\frac{\text { Donor }}{\text { transition } 1 s\left(A_{1}\right) \rightarrow}$} & \multirow{2}{*}{$\begin{array}{c}\text { Transition energy } \\
h v(\mathrm{meV})\end{array}$} & \multirow{2}{*}{$\begin{array}{l}\text { Absorption cross section } \\
\qquad \sigma(v)\left(\mathrm{cm}^{2}\right)\end{array}$} & \multicolumn{2}{|r|}{ Oscillator strength } & \multirow{2}{*}{$\begin{array}{l}\text { Calibration factor } \\
\qquad \sigma_{i}^{-1}\left(\mathrm{~cm}^{-1}\right)\end{array}$} \\
\hline & & & & Experiment & Calculated in the $D D$ model & \\
\hline \multirow[t]{8}{*}{$\mathrm{Mg}$} & $2 p_{0}$ & 95.82 & $4.38(25) \times 10^{-15}$ & $0.00652(13)$ & 0.0291 & $1.61(05) \times 10^{14}$ \\
\hline & $2 p_{ \pm}$ & 101.08 & $1.29(15) \times 10^{-14}$ & $0.01614(73)$ & 0.0604 & $7.18(09) \times 10^{13}$ \\
\hline & $3 p_{0}$ & 101.97 & $1.20(09) \times 10^{-15}$ & $0.0020(2)$ & 0.0080 & \\
\hline & $4 p_{0}(!)$ & 104.18 & $2.33(16) \times 10^{-16}$ & $0.00134(7)$ & & \\
\hline & $3 p_{ \pm}$ & 104.39 & $3.87(36) \times 10^{-15}$ & $0.0052(5)$ & 0.020 & $1.90(02) \times 10^{14}$ \\
\hline & $4 p_{ \pm}$ & 105.34 & $1.44(13) \times 10^{-15}$ & $0.00137(11)$ & & \\
\hline & $4 f_{ \pm}$ & 105.61 & $2.10(18) \times 10^{-16}$ & $0.00016(9)$ & & \\
\hline & $5 p_{ \pm}$ & 106.06 & $5.42(53) \times 10^{-16}$ & $0.00036(4)$ & & \\
\hline
\end{tabular}

TABLE II. Absorption cross sections and oscillator strengths for most intense intracenter transitions of neutral Se double donor in silicon. The transition energy and cross-section values are averaged over the investigated samples, the transition $\alpha L<1$.

\begin{tabular}{|c|c|c|c|c|c|c|}
\hline \multirow[b]{2}{*}{ Donor } & \multirow{2}{*}{$\frac{\text { Donor }}{\text { transition } 1 s\left(A_{1}\right) \rightarrow}$} & \multirow{2}{*}{$\begin{array}{c}\text { Transition energy } \\
h v(\mathrm{meV})\end{array}$} & \multirow{2}{*}{$\begin{array}{l}\text { Absorption cross } \\
\text { section } \sigma(v)\left(\mathrm{cm}^{2}\right)\end{array}$} & \multicolumn{2}{|c|}{ Oscillator strength } & \multirow{2}{*}{$\begin{array}{l}\text { Calibration factor } \\
\qquad \sigma_{i}^{-1}\left(\mathrm{~cm}^{-1}\right)\end{array}$} \\
\hline & & & & Experiment & Calculated in the $D D$ model & \\
\hline \multirow[t]{8}{*}{$\mathrm{Se}$} & $1 s\left(T_{2}\right)$ & 272.21 & $1.55(22) \times 10^{-16}$ & $0.000237(29)$ & & \\
\hline & $2 p_{0}$ & 295.17 & $1.53(28) \times 10^{-16}$ & $0.000311(24)$ & 0.00684 & $4.06(19) \times 10^{15}$ \\
\hline & $2 p_{ \pm}$ & 300.28 & $3.84(63) \times 10^{-16}$ & $0.000797(50)$ & 0.01079 & $1.49(07) \times 10^{15}$ \\
\hline & $3 p_{0}$ & 301.19 & $5.43(64) \times 10^{-17}$ & $0.000065(8)$ & 0.00215 & \\
\hline & $4 p_{0}$ & 303.37 & $2.05(40) \times 10^{-17}$ & $0.000013(2)$ & & \\
\hline & $3 p_{ \pm}$ & 303.56 & $1.02(15) \times 10^{-16}$ & $0.000234(14)$ & 0.0035 & $5.74(57) \times 10^{15}$ \\
\hline & $4 p_{ \pm}$ & 304.49 & $2.65(44) \times 10^{-17}$ & $0.000045(5)$ & & \\
\hline & $5 p_{ \pm}$ & 305.22 & $9 \times 10^{-18}$ & 0.000007 & & \\
\hline
\end{tabular}

TABLE III. Absorption cross sections, oscillator strengths for most intense intracenter transitions of neutral $\mathrm{Se}_{2}$ double donor in silicon. The transition energy and cross-section values are averaged over the investigated samples, the transition $\alpha L<0.6$.

\begin{tabular}{|c|c|c|c|c|c|}
\hline Donor & $\frac{\text { Donor }}{\text { transition } 1 s\left(A_{1}\right) \rightarrow}$ & $\begin{array}{c}\text { Transition } \\
\text { energy } h v(\mathrm{meV})\end{array}$ & $\begin{array}{l}\text { Absorption cross } \\
\text { section } \sigma(v)\left(\mathrm{cm}^{2}\right)\end{array}$ & $\begin{array}{l}\text { Oscillator strength } \\
\text { (Experiment) }\end{array}$ & $\begin{array}{c}\text { Calibration factor } \\
\sigma_{i}^{-1}\left(\mathrm{~cm}^{-1}\right)\end{array}$ \\
\hline \multirow[t]{8}{*}{$\mathrm{Se}_{2}$} & $1 s\left(E^{-}\right)$ & 175.15 & $2.27(63) \times 10^{-16}$ & $0.00034(10)$ & \\
\hline & $1 s\left(A_{1}^{-}\right)$ & 180.74 & $2.48(99) \times 10^{-16}$ & $0.000429(66)$ & \\
\hline & $2 p_{0}$ & 194.91 & $3.89(79) \times 10^{-16}$ & $0.00123(14)$ & $6.59(44) \times 10^{14}$ \\
\hline & $2 p_{ \pm}$ & 200.09 & $1.04(24) \times 10^{-15}$ & $0.00365(24)$ & $2.60(18) \times 10^{14}$ \\
\hline & $3 p_{0}$ & 200.98 & $1.58(46) \times 10^{-16}$ & $0.000233(64)$ & \\
\hline & $3 p_{ \pm}$ & 203.35 & $2.20(59) \times 10^{-16}$ & $0.000234(14)$ & $1.66(36) \times 10^{15}$ \\
\hline & $4 p_{ \pm}$ & 204.30 & $6.5(2.1) \times 10^{-17}$ & $0.000128(46)$ & \\
\hline & $5 p_{ \pm}$ & 205.04 & $1.65(25) \times 10^{-17}$ & $0.000025(2)$ & \\
\hline
\end{tabular}

TABLE IV. Absorption cross sections, oscillator strengths for most intense intracenter transitions of neutral S double donor in silicon. The transition energy and cross-section values are averaged over the investigated samples, the transition $\alpha L<0.2$.

\begin{tabular}{|c|c|c|c|c|c|c|}
\hline \multirow[b]{2}{*}{ Donor } & \multirow{2}{*}{$\frac{\text { Donor }}{\text { transition } 1 s\left(A_{1}\right) \rightarrow}$} & \multirow{2}{*}{$\begin{array}{c}\text { Transition energy } \\
h v(\mathrm{meV})\end{array}$} & \multirow{2}{*}{$\begin{array}{l}\text { Absorption cross } \\
\text { section } \sigma(v)\left(\mathrm{cm}^{2}\right)\end{array}$} & \multicolumn{2}{|c|}{ Oscillator strength } & \multirow{2}{*}{$\begin{array}{c}\text { Calibration factor } \\
\qquad \sigma_{i}^{-1}\left(\mathrm{~cm}^{-1}\right)\end{array}$} \\
\hline & & & & Experiment & Calculated in the $D D$ model & \\
\hline \multirow[t]{7}{*}{$\mathrm{S}$} & $1 s\left(T_{2}\right)$ & 283.72 & $4.76(81) \times 10^{-16}$ & $0.000283(19)$ & & \\
\hline & $2 p_{0}$ & 306.86 & $1.78(23) \times 10^{-16}$ & $0.000234(8)$ & 0.00644 & $4.33(15) \times 10^{15}$ \\
\hline & $2 p_{ \pm}$ & 311.94 & $4.27(92) \times 10^{-16}$ & $0.000639(28)$ & 0.01009 & $1.50(07) \times 10^{15}$ \\
\hline & $3 p_{0}$ & 312.85 & $4.95(27) \times 10^{-17}$ & $0.000087(4)$ & 0.00207 & \\
\hline & $4 p_{0}$ & 315.02 & $2.46(68) \times 10^{-17}$ & $0.000012(2)$ & & \\
\hline & $3 p_{ \pm}$ & 315.21 & $2.15(27) \times 10^{-16}$ & $0.000195(11)$ & 0.0035 & $5.57(46) \times 10^{15}$ \\
\hline & $4 p_{ \pm}$ & 316.15 & $3.31(36) \times 10^{-17}$ & $0.000039(3)$ & & \\
\hline
\end{tabular}


TABLE V. Absorption cross sections, oscillator strengths for most intense intracenter transitions of neutral $S_{2}$ double donor in silicon. The transition energy and cross-section values are averaged over the investigated samples with the transition $\alpha L<1$.

\begin{tabular}{|c|c|c|c|c|c|}
\hline Donor & $\frac{\text { Donor }}{\text { transition } 1 s\left(A_{1}\right) \rightarrow}$ & $\begin{array}{c}\text { Transition energy } \\
h v(\mathrm{meV})\end{array}$ & $\begin{array}{l}\text { Absorption cross } \\
\text { section } \sigma(v)\left(\mathrm{cm}^{2}\right)\end{array}$ & $\begin{array}{l}\text { Oscillator strength } \\
\text { (Experiment) }\end{array}$ & $\begin{array}{c}\text { Calibration factor } \\
\sigma_{i}^{-1}\left(\mathrm{~cm}^{-1}\right)\end{array}$ \\
\hline \multirow[t]{7}{*}{$S_{2}$} & $1 s\left(E^{-}\right)$ & 161.12 & $1.19(15) \times 10^{-16}$ & $0.000218(48)$ & \\
\hline & $1 s\left(A_{1}^{-}\right)$ & 156.37 & $1.22(16) \times 10^{-16}$ & $0.000149(21)$ & \\
\hline & $2 p_{0}$ & 176.13 & $3.86(51) \times 10^{-16}$ & $0.001170(35)$ & $1.07(13) \times 10^{15}$ \\
\hline & $2 p_{ \pm}$ & 181.24 & $1.09(13) \times 10^{-15}$ & $0.00319(12)$ & $4.11(54) \times 10^{14}$ \\
\hline & $3 p_{0}$ & 182.13 & $9.82(1.76) \times 10^{-17}$ & $0.000153(29)$ & \\
\hline & $3 p_{ \pm}$ & 184.51 & $3.97(1.09) \times 10^{-16}$ & $0.00075(17)$ & $2.25(40) \times 10^{15}$ \\
\hline & $4 p_{ \pm}$ & 185.46 & $1.47(29) \times 10^{-16}$ & $0.000218(39)$ & \\
\hline
\end{tabular}

in close vicinity with the linearly dependent (on concentrations) integrated absorptions, while the samples from crystals with closer values of both donor types exhibit stronger scattering around this main trend. From the analysis of the errors in the calibration factors and related parameters for double donors, the determination of concentrations by modeling Hall $n$ (T) dependences was found to have the main impact, as expected from the beginning of this study.

We note that the strongest deviation from the mean values of oscillator strengths and calibration factors are observed for thinner samples. We hypothesize that this is due to the stronger impact from the multiple reflections inside thinner samples leading to a slight enhancement of the transmitted light onto the detector in comparison with the influence of such a factor for thicker samples. For this reason, these stronger deviated values were omitted in the averaging procedure while obtaining mean values shown in Tables I-V.

\section{CALCULATION OF OSCILLATOR STRENGTHS FOR INTRACENTER TRANSITIONS OF A DOUBLE DONOR IN Si}

The oscillator strengths of dipole intracenter transitions for single-electron substitutional impurities in silicon were commonly calculated in the effective mass approximation [18]. In this approach, the central-cell correction was evaluated through the variation of a point-charge potential to adjust the calculated energy of the ground donor state to the experimental values of binding energies. In this way, calculated oscillator strengths were found to be very close to the reported values derived from infrared absorption spectroscopy of samples with different shallow impurities (Fig. 5).

Such an approach, however, cannot be directly used to describe the center with two excess electrons. We proposed a simplified model of a two-electron impurity center in silicon for calculations of state eigen wave functions and strengths of optical intracenter transitions, for further details see the Supplemental Material [23]. In this model, the states of twoelectron impurities are divided into two groups: spin-singlet (para-states) and spin-triplet (ortho-states) states, like in a helium atom [38]. The ground state of a two-electron atom is a para state. The interaction between electrons and nucleus is reduced due to partial screening of the nucleus potential by one ("inner") of two bound electrons, while the second ("outer") electron moves in a static potential of a partially screened atomic nucleus. Assuming different radii of the orbitals of the inner and outer electrons, wave functions of excited states of the outer electron are constructed from hydrogenlike wave functions when the Coulomb potential is formed by the charge (a)

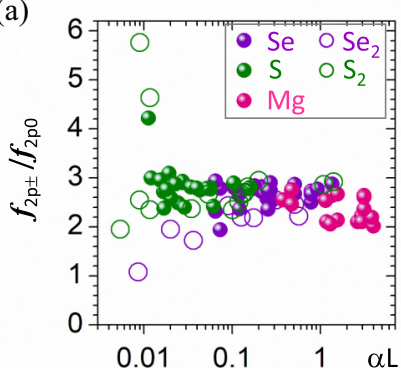

(c)
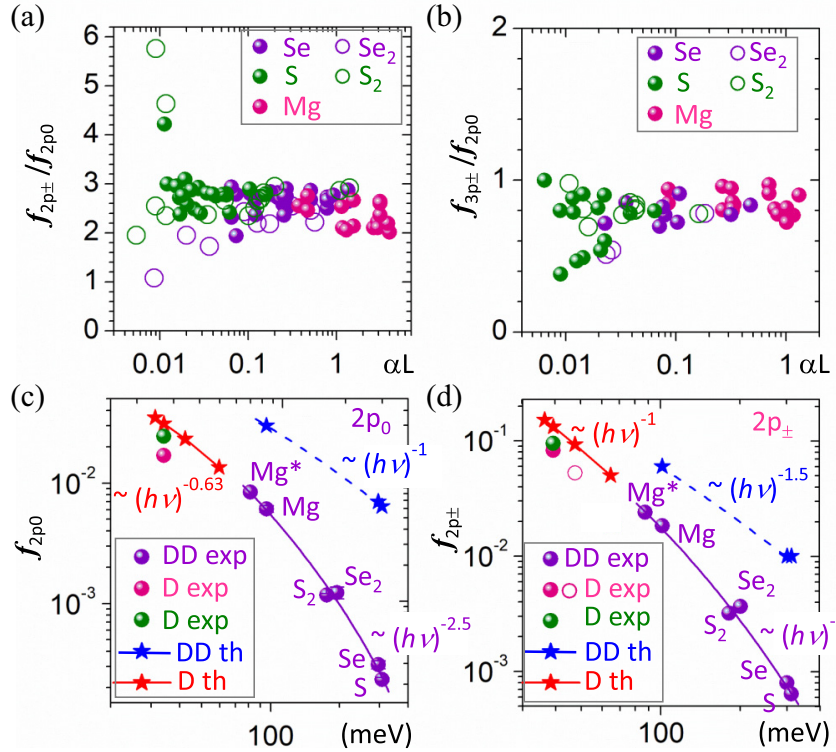

(e)
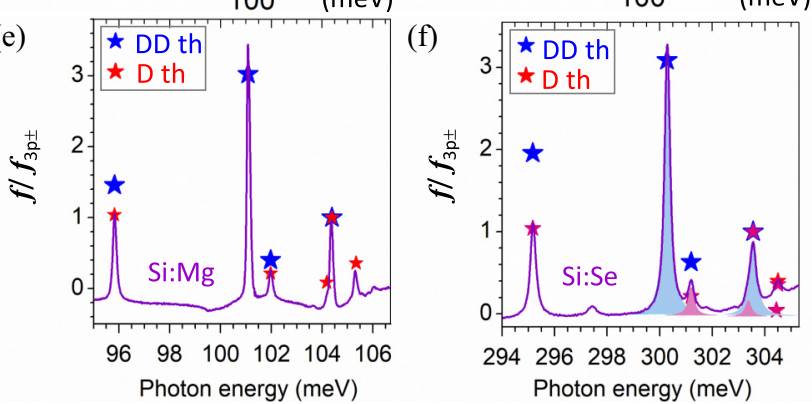

FIG. 5. Experimental (exp) and theoretical (th) dependences of oscillator strengths and their ratios for most intense intracenter transitions (from the ground center state into $2 p_{0}, 2 p_{ \pm}$and $3 p_{ \pm}$states) of double $(D D)$ and single $(D)$ donors in Si: (a), (b) on optical density of samples, as well as on the transition energy (c)-(f), in comparison with the transition intensities in absorption spectra of Si:Mg no. 158 (e) and Si:Se no. 42-10a (f) samples. Experimental data for single donors [D exp in (c), (d)]: pink (open and hollow) are from Refs. [41,42], respectively; green - this work. Lorentzian fits for the overlapping transitions pairs (the lower energy into the $2 p_{ \pm}$ and $3 p_{0}$ states and the higher energy of $4 p_{0}$ and $3 p_{ \pm}$) are shown. 
TABLE VI. Transition oscillator strength ratios for single (SD) and double $(D D)$ donors in silicon. Transition energies for the $S D$ centers are taken from Ref. [39].

\begin{tabular}{|c|c|c|c|c|c|c|c|c|}
\hline \multirow{2}{*}{$\frac{f}{\text { ratio }}$} & \multirow[b]{2}{*}{$D D$} & \multirow[b]{2}{*}{$h v$ ratio } & \multicolumn{2}{|c|}{$f$ ratio, $D D$} & \multirow[b]{2}{*}{$S D$} & \multirow[b]{2}{*}{$h v$ ratio } & \multicolumn{2}{|c|}{$f$ ratio, $S D$} \\
\hline & & & Exp. & Cal. & & & Exp. & Calculated [18] \\
\hline \multirow{10}{*}{$\begin{array}{l}f_{2 \mathrm{p} \pm} / \\
f_{2 \mathrm{p} 0}\end{array}$} & $\mathrm{Mg}$ & 1.055 & $2.48(7)$ & 2.078 & EMT & 1.26 & & $4.91,12.39$ [17] \\
\hline & & & $2.14[40]$ & & $\mathrm{Sb}$ & 1.164 & & $4.34,11.74[17]$ \\
\hline & $S_{2}$ & 1.029 & $2.62(6)$ & & $\mathrm{P}$ & 1.148 & $3.57[14]$ & $4.25,10.16[17]$ \\
\hline & $\mathrm{Se}_{2}$ & 1.026 & $2.68(13)$ & & & & $3.67[15]$ & \\
\hline & $\mathrm{Se}$ & 1.017 & $2.69(3)$ & 1.578 & & & $4.39[41]$ & \\
\hline & $\mathrm{S}$ & 1.017 & $2.76(4)$ & 1.566 & & & $>4.3[42]$ & \\
\hline & & & & & As & 1.121 & & $4.00,9.71[17]$ \\
\hline & & & & & $\mathrm{Bi}$ & 1.085 & & 3.72 \\
\hline & $\mathrm{Mg}$ & 1.089 & $0.80(4)$ & 0.687 & EMT & 1.42 & & $0.936,2.16[17]$ \\
\hline & $S_{2}$ & 1.045 & $0.79(2)$ & & $\mathrm{Sb}$ & 1.173 & & $0.96,2.12[17]$ \\
\hline \multirow{3}{*}{$\begin{array}{l}f_{3 \mathrm{p} \pm} / \\
f_{2 \mathrm{p} 0}\end{array}$} & $\mathrm{Se}_{2}$ & 1.043 & $0.65(5)$ & & $\mathrm{P}$ & 1.244 & $0.973[42]$ & $0.961,2.02$ [17] \\
\hline & $\mathrm{Se}$ & 1.028 & $0.74(3)$ & 0.512 & As & 1.198 & & $0.965,1.97[17]$ \\
\hline & $\mathrm{S}$ & 1.027 & $0.79(4)$ & 0.543 & $\mathrm{Bi}$ & 1.140 & & 0.948 \\
\hline \multirow{7}{*}{$\begin{array}{l}f_{3 \mathrm{p} 0} / \\
f_{2 \mathrm{p} 0}\end{array}$} & & & & & EMT & 1.30 & & 0.101 [17], 0.138 \\
\hline & $\mathrm{Mg}$ & 1.064 & $0.30(2)$ & 0.273 & $\mathrm{Sb}$ & 1.193 & & 0.101 [17], 0.197 \\
\hline & $S_{2}$ & 1.034 & $0.28(1)$ & & $\mathrm{P}$ & 1.176 & 0.19 [14] & 0.174 [17], 0.205 \\
\hline & $\mathrm{Se}_{2}$ & 1.031 & $0.30(5)$ & & & & $0.19[15]$ & \\
\hline & $\mathrm{Se}$ & 1.020 & $0.27(2)$ & 0.315 & As & 1.142 & & 0.195 [17], 0.227 \\
\hline & $\mathrm{S}$ & 1.020 & $0.31(5)$ & 0.321 & $\mathrm{Bi}$ & 1.100 & & 0.259 \\
\hline & $\mathrm{Mg}$ & 1.032 & $0.32(5)$ & 0.330 & EMT & 1.13 & & 0.174 [17], 0.191 \\
\hline \multirow{4}{*}{$\begin{array}{l}f_{3 \mathrm{p} \pm} / \\
f_{2 \mathrm{p} \pm}\end{array}$} & $S_{2}$ & 1.017 & $0.30(5)$ & & $\mathrm{Sb}$ & 1.090 & & 0.180 [17], 0.221 \\
\hline & $\mathrm{Se}_{2}$ & 1.016 & $0.24(11)$ & & $\mathrm{P}$ & 1.083 & $<0.22[42]$ & 0.198 [17], 0.226 \\
\hline & $\mathrm{Se}$ & 1.011 & $0.28(4)$ & 0.324 & As & 1.069 & & 0.203 [17], 0.241 \\
\hline & $\mathrm{S}$ & 1.010 & $0.29(4)$ & 0.347 & $\mathrm{Bi}$ & 1.051 & & 0.255 \\
\hline
\end{tabular}

+1 , while the inner electron is not affected by interaction with the outer electron and virtually moves in the Coulomb potential of the +2 charged ion. For the ground state, the screening of the nuclear potential by an electron is partial, and each electron moves in a Coulomb potential formed by a fractional charge.

The oscillator strengths were calculated then using formalism like the case of group- $\mathrm{V}$ donors in silicon and germanium [18]. Oscillator strengths were calculated for a few most intense dipole-allowed intracenter transitions, characterized by the largest deviations from the values of the EMT.

\section{DISCUSSION}

While double donors in silicon exhibit spectra of excited states that are similar in general to those for the single donors, their intracenter transition strengths appear to be quantitatively different, much more intensively decaying with the center chemical shift (i.e., also with the binding energy $E_{\mathrm{DD}}$ ) than the $D D$ theoretical predictions.

The $D D$ approach, proposed in this work, gives a good prediction of the relative $f$ for the impurity transitions terminating in the similar type $\left(n p_{0}\right.$ vs $\left.n p_{0}\right)$ state and reasonable values for those terminating in the states with different types $\left(n p_{ \pm}\right.$vs $\left.n p_{0}\right)$ of the wave functions [Table VI, Fig. 5(e)]. The predicted absolute $f$ values for the selected $D D$ transitions differ significantly from the experiment. We stress here that $f$ depends strongly on a choice of the parameters determining anisotropy of the $D D$ state wave function, which can be further adjusted towards empiric values of $D D$ spatial localization. However, this simplified model of a $D D$ center was aimed mainly to find if the approach predicts the trends on the relative strength of the $D D$ intracenter transitions terminating in the states with the similar type of wave function $\left(n p_{0} \mathrm{vs} n p_{0}\right)$ and in the different types $\left(n p_{ \pm} \mathrm{vs} n p_{0}\right)$ as well as the $f$ dependences on intracenter transition energy, and it has showed reasonable agreement with the experimental data in both these aspects.

When compared with the single donor $(S D)$ centers in silicon, the theory predicts a slight divergence for the binding energy $E_{\mathrm{SD}}$ oscillator strengths of hydrogenlike donors that reflects the weakly nonlinear drop of $f$ with an increase of $E_{\mathrm{SD}}$ (Table VI). Although some $S D$ theories deliver $f$ values reasonably close to the observed experimental values for particular $S D$ intracenter transitions, the ratios of $f$ for the transitions terminating in the similar $\left(n p_{0} v s n p_{0}\right)$ and in the different types ( $n p_{ \pm}$vs $n p_{0}$ ) of wave functions differ more in comparison with the experiment, which may point to either the quality of the model wave functions for the excited states of $S D$ or on strong dependences of $f$ on concentration of donors or other abundant defects.

All theories underestimate the power law of $f$ dependences on the transition energies (Table VI), Figs. 5(c) and 5(d). The ratio between oscillator strengths of most intense transitions of $D D$ in the lowest excited odd-parity states, $2 p_{0}, 2 p_{ \pm}$, and $3 p_{ \pm}$, remains approximately the same through the $D D$ type, if the absorbance at the transition is studied within moderate 
optical density, i.e., in the reliable range of $\mathrm{OD}=[0.01 ; 1]$, Figs. 5(a) and 5(b).

Analysis of discrepancies between theoretically obtained values of oscillator strengths for various donors in $\mathrm{Si}$ and $\mathrm{Ge}$, calculated within the framework of the effective mass, and known experimental values shows that the deviation is larger for the larger binding energy of the impurity center. These deviations are associated with incorrect consideration of the short-range potential of the impurity center, which has a greater weight in the total potential of ionized impurity for the deeper donors. The difference between the oscillator strengths calculated in the present work for two-electron donors and the measured values has obviously the same reason as for the single-electron centers. This allows us to conclude the presence of a short-range potential in the electron-ion interaction, (which is challenging for a universal theoretical description) as the main reason, while the features of the structure of the wave function caused by the interaction of two electrons have less impact for this discrepancy.

\section{CONCLUSIONS}

Infrared absorption cross sections, calibration factors, and oscillator strengths of several intracenter transitions of interstitial magnesium and substitutional chalcogen double donors in silicon were determined by analyzing a large number of crystals differently doped by diffusion techniques. Temperature-dependent Hall effect measurements were used to derive concentrations of electrically active donor centers abundant in the investigated samples and having different thermal activation energies. Low values of oscillator strengths for intracenter transitions of deep centers enabled such characterization in the broad range of concentrations, above two orders of magnitude, which is not feasible for single-electron impurity centers. Despite the error bars introduced by inaccuracy in the values of concentrations of deep donors as it is determined by modeling the Hall dependences of free electron density, such a technique provided a unique possibility for quantitative transformation of concentration values into the optical characterization for double donors. Within the accuracy of determination of partial concentrations of different types of double donors in silicon, the mean values of the calibration factors and transition oscillation strengths of investigated impurities, obtained by averaging over the whole set of studied samples, give the same values for various initial silicon crystals, different diffusion conditions, and postdiffusion treatments of the samples. The oscillator strengths of double donors obey much stronger dependences on intracenter transitions energies than those predicted and partially observed for the single-electron donor centers in silicon. This indicates that it is necessary to include a short-range potential description for calculation of the accurate wave function of the donor states, presumably mostly expressed for the double donor ground state. The validity of the effective-mass-based approaches for appropriate description of such deep centers is broken - even with an attempt to adapt such an approach by introducing an anisotropy in the ground-state wave function, as undertaken in this work.

As an additional outcome of this study, calibration factors for double donors $\mathrm{Mg}$, Se, and $\mathrm{S}$ were obtained, which can be of practical importance for an optical characterization of deep donors in silicon.

\section{ACKNOWLEDGMENTS}

Research carried out at the Ioffe Institute was done within the framework of the state assignment to the Ioffe Institute. S.A.L. thanks the Leverhulme Trust for partially funding this work under Research Project Grant RPG-2014-262, “Quantum optics of mid gap chalcogen donors in single crystal silicon".
[1] N. Q. Vinh, P. T. Greenland, K. Litvinenko, B. Redlich, A. F. G. van der Meer, S. A. Lynch, M. Warner, A. M. Stoneham, G. Aeppli, D. J. Paul, C. R. Pidgeon, and B. N. Murdin, Proc. Natl. Acad. Sci. USA 105, 10649 (2008).

[2] D. Karaiskaj, J. A. H. Stotz, T. Meyer, M. L. W. Thewalt, and M. Cardona, Phys. Rev. Lett. 90, 186402 (2003).

[3] B. E. Kane, Nature (London) 393, 133 (1998).

[4] K. J. Morse, R. J. S. Abraham, A. DeAbreu, C. Bowness, T. S. Richards, H. Riemann, N. V. Abrosimov, P. Becker, H.-J. Pohl, M. L. W. Thewalt, and S. Simmons, Sci. Adv. 3, e1700930 (2017).

[5] E. Janzén, R. Stedman, G. Grossmann, and H. G. Grimmeiss, Phys. Rev. B 29, 1907 (1984).

[6] A. DeAbreu, C. Bowness, R. J. S. Abraham, A. Medvedova, K. J. Morse, H. Riemann, N. V. Abrosimov, P. Becker, H.-J. Pohl, M. L. W. Thewalt, and S. Simmons, Phys. Rev. Appl. 11, 044036 (2019).

[7] S. G. Pavlov, Yu. A. Astrov, L. M. Portsel, V. B. Shuman, A. N. Lodygin, N. V. Abrosimov, and H.-W. Hübers, Mater. Sci. Semicond. Process. 130, 105833 (2021).
[8] P. B. Coates, Metrologia 17, 103 (1981).

[9] V. M. Marchenko, H.-G. Purwins, L. M. Portsel, and Yu. A. Astrov, Semiconductor-Gas-Discharge Device for Fast Imaging in the Infrared: Physics, Engineering and Applications (Shaker Verlag, Aachen, 2016).

[10] N. Sclar, Infrared Phys. 17, 71 (1977).

[11] C. B. Simmons, A. J. Akey, J. P. Mailoa, D. Recht, M. J. Aziz, and T. Buonassisi, Adv. Funct. Mater. 24, 2852 (2014).

[12] R. Fain, S. A. Miller, M. Yu, A. G. Griffith, J. Cardenas, and M. Lipson, OSA Technical Digest (online) (Optical Society of America, 2017), CLEO: Science and Innovations 2017, San Jose, CA, USA, 14-19 May 2017, paper STu1N.4.

[13] E. Rotsaert, P. Clauws, J. Vennik, and L. Van Goethem, J. Appl. Phys. 65, 730 (1989).

[14] H. Ch. Alt, M. Gellon, M. G. Pretto, R. Scala, F. Bittersberger, K. Hesse, and A. Kempf, AIP Conf. Proc. 449, 201 (1998).

[15] M. Porrini, M. G. Pretto, R. Scala, A. V. Batunina, H. C. Alt, and R. Wolf, Appl. Phys. A 81, 1187 (2005). 
[16] A. T. Collins and A. W. S. Williams, J. Phys. C Solid State Phys. 4, 1789 (1971).

[17] I. L. Beĭnikhes and Sh. M. Kogan, JETP 66, 164 (1987) [Zh. Eksp. Teor. Fiz. 93, 285 (1987) (in Russian)].

[18] P. Clauws, J. Broeckx, E. Rotsaert, and J. Vennik, Phys. Rev. B 38, 12377 (1988).

[19] R. W. Jansen and O. F. Sankey, Phys. Rev. B 33, 3994 (1986).

[20] S. G. Pavlov, D. L. Kamenskyi, Yu. A. Astrov, V. B. Shuman, L. M. Portsel, A. N. Lodygin, N. V. Abrosimov, H. Engelkamp, A. Marchese, and H.-W. Hübers, Phys. Rev. B 102, 115205 (2020).

[21] R. J. S. Abraham, A. DeAbreu, K. J. Morse, V. B. Shuman, L. M. Portsel, A. N. Lodygin, Yu. A. Astrov, N. V. Abrosimov, S. G. Pavlov, H.-W. Hübers, S. Simmons, and M. L. W. Thewalt, Phys. Rev. B 98, 045202 (2018).

[22] V. N. Shastin, R. Kh. Zhukavin, K. A. Kovalevsky, V. V. Tsyplenkov, V. V. Rumyantsev, D. V. Shengurov, S. G. Pavlov, V. B. Shuman, L. M. Portsel, A. N. Lodygin, Yu. A. Astrov, N. V. Abrosimov, J. M. Klopf, and H.-W. Hübers, Semiconductors 53, 1234 (2019).

[23] See Supplemental Material at http://link.aps.org/supplemental/ 10.1103/PhysRevMaterials.5.114607 for details of preparation and parameters of the samples, details of calibration of absorption spectra and description of statistical approach for determination of partial concentrations of double donors, as well as calculation of oscillator strengths for intracenter transitions of a double donor in silicon.

[24] Yu. A. Astrov, L. M. Portsel, A. N. Lodygin, and V. B. Shuman, Semicond. Sci. Technol. 26, 055021 (2011).

[25] Yu. A. Astrov, V. B. Shuman, L. M. Portsel, and A. N. Lodygin, Semiconductors 48, 413 (2014).

[26] Yu. A. Astrov, S. A. Lynch, V. B. Shuman, L. M. Portsel, A. A. Makhova, and A. N. Lodygin, Semiconductors 47, 247 (2013).
[27] V. B. Shuman, L. M. Portsel, A. N. Lodygin, and Yu. A. Astrov, Semiconductors 49, 421 (2015).

[28] R. K. Franks and J. B. Robertson, Solid State Commun. 5, 479 (1967).

[29] V. B. Shuman, Yu. A. Astrov, A. N. Lodygin, and L. M. Portsel, Semiconductors 51, 1031 (2017).

[30] Yu. A. Astrov, V. B. Shuman, L. M. Portsel, A. N. Lodygin, S. G. Pavlov, N. V. Abrosimov, V. N. Shastin, and H.-W. Hübers, Phys. Stat. Solidi A 214, 1700192 (2017).

[31] W. R. Thurber, R. L. Mattis, Y. M. Liu, and J. J. Filliben, National Bureau of Standards Special Publication 400 (1981), Table 10, p. 34, and Table 14, p. 40.

[32] L. T. Ho, Phys. Status Solidi 210, 313 (1998).

[33] E. V. Loewenstein, D. R. Smith, and R. L. Morgan, Appl. Opt. 12, 398 (1973).

[34] B. J. Frey, D. B. Leviton, and T. J. Madison, Proc. SPIE 6273, Optomechanical Technologies for Astronomy, 62732J, edited by Eli Atad-Ettedgui, J. Antebi, and D. Lemke (6 July 2006). Event: SPIE Astronomical Telescopes + Instrumentation, 24 31 May 2006, Orlando, FL, USA.

[35] F. A. Johnson, Proc. Phys. Soc. 73, 265 (1959).

[36] D. L. Dexter, Solid State Phys. 6, 355 (1958).

[37] R. C. Hilborn, Am. J. Phys. 50, 982 (1982).

[38] H. A. Bethe and E. E. Salpeter, Quantum Mechanics of Oneand Two-Electron Atoms (Springer-Verlag, Berlin Göttingen Heidelberg, 1957).

[39] B. Pajot, Optical Absorption of Impurities and Defects in Semiconducting Crystals: I. Hydrogen-Like Centres (Springer, Berlin, 2010).

[40] Yu. A. Astrov, L. M. Portsel, V. B. Shuman, A. N. Lodygin, N. V. Abrosimov, S. G. Pavlov, and H.-W. Hübers, Phys. Techn. Semicond. 55, 299 (2021) (in Russian).

[41] H. Shirai and Y. Yanase, Appl. Phys. A 120, 927 (2015).

[42] B. A. Andreev, Mater. Sci. Forum 196-201, 121 (1995). 